\title{
Driver Training Simulator for Backing Up Commercial Vehicles with Trailers*
}

\author{
Uwe BERG**, Philipp WOJKE** and Dieter ZÖBEL** \\ ${ }^{* *}$ Department of Computer Science, University Koblenz-Landau \\ Universitätsstraße 1, D-56070 Koblenz, Germany \\ E-mail: uwe.berg@uni-koblenz.de
}

\begin{abstract}
Backing up tractors with trailers is a difficult task since the kinematic behavior of articulated vehicles is complex and hard to control. Especially unskilled drivers are overstrained with the complicated steering process. To learn and practice the steering behavior of articulated vehicles, we developed a 3D driving simulator. The simulator can handle different types of articulated vehicles like semi-trailers, oneand two-axle trailers, or gigaliners. The use of a driving simulator offers many advantages over the use of real vehicles. One of the main advantages is the possibility to learn the steering behavior of all vehicle types. Drivers can be given more and better driving instructions like collision warnings or steering hints. Furthermore, the driver training costs can be reduced. Moreover, mistakes of the student do not lead to real damages and costly repairs. The hardware of the simulator consists of a low cost commercial driving stand with original truck parts, a projection of the windshield and two flat panel monitors for the left and right exterior mirrors. Standard PC hardware is used for controlling the driving stand and for generating the realtime 3D environment. Each aspect of the simulation like realistic vehicle movements or generation of different views, is handled by a specific software module. This flexible system can be easily extended which offers the opportunity for other uses than just driver training. Therefore, we use the simulator for the development and test of driver assistance systems.
\end{abstract}

Key words: Driving Simulator, Driver Training, Backing Up, Reversing, Articulated Vehicles, Tractor-Trailer System, Kinematics

\section{Introduction}

Backing up articulated vehicles is accompanied by a high degree of difficulty. The kinematic behavior of this class of vehicles is very complex and hard to control. In particular, unskilled drivers like novice drivers are in general overstrained with the complicated steering process. There are four main reasons causing difficulties in backing up articulated vehicles:

- Unfamiliar steering process

- Illogical steering process

- Restricted visibility

- Complex vehicle technology

At first, the steering process to reverse a car or an articulated vehicle is often unfamiliar to the driver. The time used for this kind of driving maneuvers in relation to the whole driving time is marginal. Therefore, many drivers have only slight experience in backing up vehicles. Another important reason is that the driver can be faced with a situation, which initially requires an illogical steering angle. Assume that the driver wants to back up a truck 
with one-axle trailer in a left turn and truck and trailer are directly straightened. At first, he/she has to turn the steering wheel to the right side. When the trailer reaches the designated direction, the truck is also steered into the primarily intended direction. This two-stage steering process is the cause for many steering mistakes. In the case of a two-axle trailer, the driver is actually faced with a three-stage steering process which is a challenge for professional truck drivers as well.

In addition, there is a restricted visibility to the area behind and alongside the vehicle (see Fig. 1). The hatched area in Fig. 1 is not visible to the driver. This means that the driver has a restricted knowledge about the environment around the articulated vehicle. He actually doesn't know, what's going on around his vehicle.

Furthermore, a risk-aggravating factor is the increasing complexity of vehicle technology. In some European countries, there are gigaliners with two trailers allowed. This kind of vehicle is said to be very hard to control during reverse driving maneuvers.

In spite of these difficulties, there are only a few scientific approaches to analyze

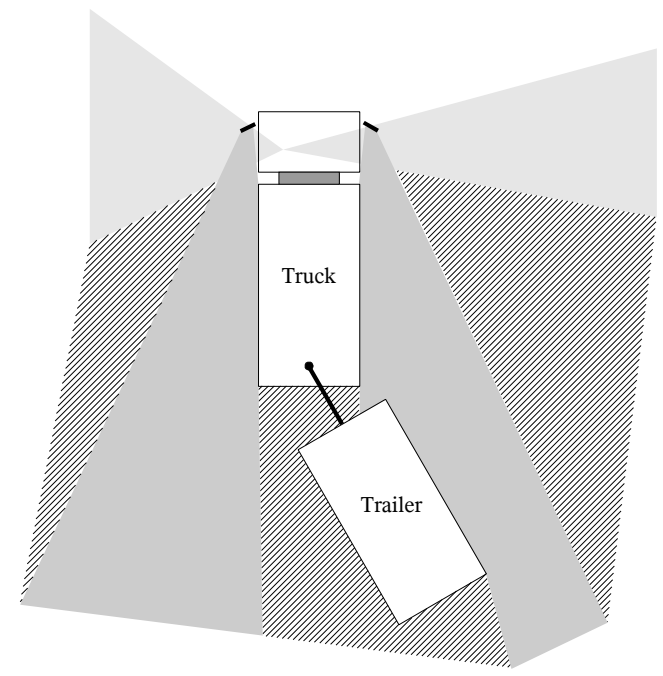

Fig. 1: Visibility of the environment around truck and trailer. the steering behavior of articulated vehicles ${ }^{(1)(2)}$ and to support student drivers or novice drivers ${ }^{(3)}$. To reduce the difficulties, we are developing steering assistance systems at the University Koblenz-Landau for backing up articulated vehicles ${ }^{(4)(5)}$. Furthermore, we are developing a non-motion truck driving simulator which can be used to study the steering behavior of trucks with one-axle trailer, semi-trailer, two-axle trailer with single-pivot steering or a gigaliner. The simulator can be used to train typical driving maneuvers like driving straight backwards or driving backwards around a 90 degree curve.

The paper starts with a brief description of the kinematics of articulated vehicles. Exemplarily some kinematic properties of a truck with one-axle trailer will be shown. Based on the kinematics, we describe the software architecture used to design the 3D-simulation. Afterwards, the simulator hardware and especially the 3D-simulation are illustrated. Furthermore, the application possibilities of the simulator are discussed. The paper closes with a depiction of our experiences in using the simulator.

\section{Nomenclature}

$$
\begin{array}{lll}
\alpha & : & \text { steering angle, } \mathrm{rad} \\
\gamma & : \text { bending angle, } \mathrm{rad} \\
\mathrm{lza} & : \text { wheel base, } \mathrm{m} \\
\text { lzk } & : \text { distance between coupling device and front axle, } \mathrm{m} \\
\text { laa } & : \text { distance between trailer axle and coupling device, } \mathrm{m} \\
\mathrm{P} & : \text { maneuver phase } \\
\mathrm{d} & : \text { distance, } \mathrm{m}
\end{array}
$$

\section{Subscripts}

$$
\begin{aligned}
& \max : \text { maximum } \\
& \text { crit }: \text { critical } \\
& \lim : \text { limited }
\end{aligned}
$$




\section{Kinematics}

There are numerous approaches to solve the problem of backing up a tractor-trailer system. A set of non-analytical techniques based on neural networks ${ }^{(6)}$, fuzzy control ${ }^{(7)}$ or genetic algorithms ${ }^{(8)}$ have been analyzed mainly for purposes of simulation. The emphasis of these solutions is on the principle solvability of the backer-upper problem by certain computational paradigms. In contrast, our approach is to describe the vehicle kinematics using an analytical approach.

At first, all vehicle movements in the driving simulation are based on the kinematics of articulated vehicles. Vehicles driving backwards are very slowly in general. Since the impact of the kinetics to slow vehicles is marginal the kinetics of vehicles has not been considered. For the analytical description of the kinematics of articulated vehicles we use the well known single track model (see Fig. 2). In this model each axle is reduced to a single wheel located in the middle of the respective axle.

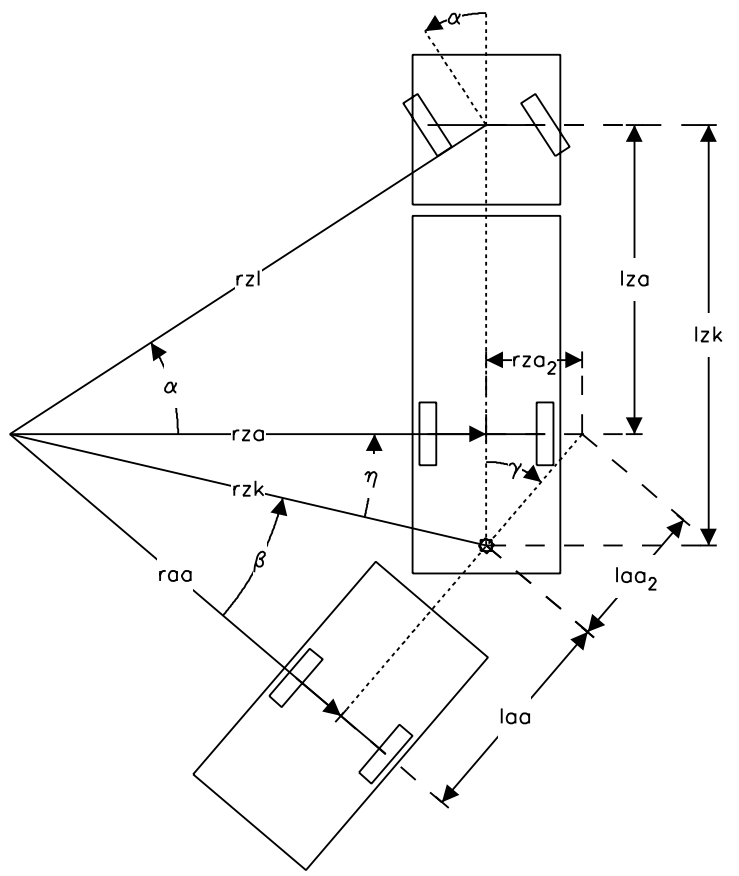

Fig. 2: Single track model of a truck with one-axle trailer.

In order to compute the kinematic behavior of an articulated vehicle, the state of the tractor and trailer must be known. This state can be described by

- the steering angle $\alpha$ (angle of the tractors front wheel) and

- the bending angle $\gamma$ (angle between the longitudinal axes of tractor and trailer).

Based on this information, some very important kinematic properties ${ }^{(4)}$ can be computed. Let us assume a truck with a one-axle trailer driving forward with an arbitrary but definite steering angle. The truck-trailer-system asymptotically approaches a configuration with a constant bending angle and truck and trailer will move on concentric circles. This state is said to be stable. If the tractor reverses with the same steering angle, the bending angle will not change. This leads to a dependency between the steering angle $\alpha$ and the bending angle $\gamma$ which can be described by the following equations:

$$
\begin{aligned}
& \alpha(\gamma)=\arctan \left(\frac{l z a * \sin (-\gamma)}{l a a+(l z k-l z a) * \cos (\gamma)}\right) \\
& \gamma(\alpha)=-\left(a \sin \left(\frac{l a a * \tan (\alpha)}{l z a * \sqrt{1+\frac{(l z a-l z k)^{2} * \tan (\alpha)}{l z a^{2}}}}\right)+a \tan \left(\frac{(l z k-l z a) * \tan (\alpha)}{l z a}\right)\right)
\end{aligned}
$$


In each real vehicle, the steering angle $\alpha$ is restricted to the interval $\left[-\alpha_{\max }, \alpha_{\max }\right]$. Based on the maximum steering angle $\alpha_{\max }$, the corresponding bending angle $\gamma_{\text {lim }}=\left|\gamma\left(\alpha_{\max }\right)\right|$ can be computed. If this characteristic bending angle $\gamma_{\text {lim }}$ is exceeded, the controllability of the articulated vehicle is limited. In this situation, tractor and trailer can only be straightened by driving forward. Therefore, the angle $\gamma_{\text {lim }}$ is called limited bending angle.

In this context, there is another particular characteristic of articulated vehicles described in Fig. 3. Assume a tractor with one-axle trailer shall be driven around a corner. Figure 3 shows the corresponding bending angle $\gamma$ and the steering angle $\alpha$ depending on the distance $\mathrm{d}$ done by the coupling device. In position $\mathrm{d}_{0}$, the vehicle starts with a bending angle $\gamma=0^{\circ}$ and a steering angle $\alpha=\alpha_{\max }$. In phase $P_{1}$, the vehicle drives backwards until position $d_{1}$ is reached. During the phase $\mathrm{P}_{2}$ the vehicle drives backwards on concentric circles. In the phase $P_{3}$, the articulated vehicle is tried to be straightened by a steering angle $\alpha=-\alpha_{\max }$. Figure 3 shows that the distance $\mathrm{d} 3-\mathrm{d} 2$ needed to straighten the articulated vehicle is much longer than the distance $\mathrm{d} 1-\mathrm{d} 0$ to bend truck and trailer to a certain angle. The more the bending angle approaches the limited value $\gamma_{\text {lim }}$ the more time is needed to straighten the articulated vehicle ${ }^{(1)}$. In consequence of this, the driver has to take care that the bending angle should fall clearly below the limited value $\gamma_{\text {lim. }}$. In practical tests, the following empirical formula has been proved to be useful:

$$
|\gamma|<0.8^{*} \gamma_{\lim }
$$

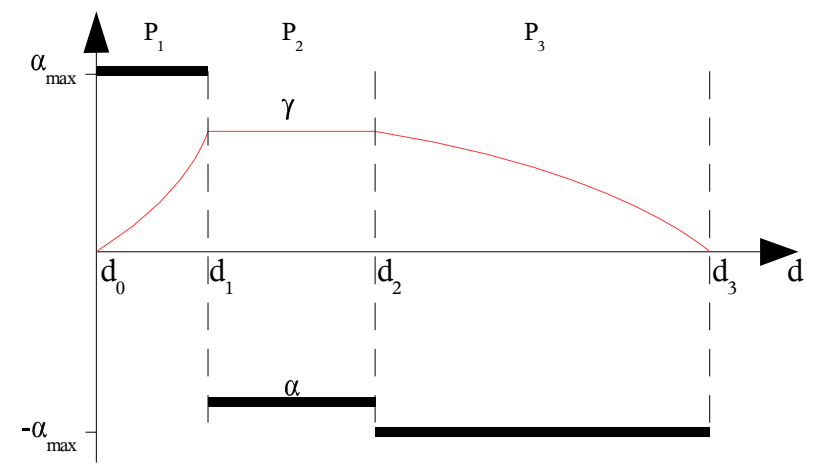

Fig. 3: Characteristics of the bending angle $\gamma$ and the steering angle $\alpha$ in dependency of the driven distance $\mathrm{d}$.

In the case of a two-axle trailer with semi-pivot steering, there are actually two limited bending angles which can be exceeded independently. Both angles can be computed on the basis of an extended single track model as well.

If the limited bending angle is exceeded, the bending angle will increase rapidly during reverse driving maneuvers. At the same time, the danger of a collision between truck and trailer increases. Therefore, the corresponding angle $\gamma_{\text {crit }}$ is called critical bending angle.

\section{Software Architecture}

Our working group mainly uses the simulator for creating, testing and evaluating different kinds of applications based on reversing articulated vehicles. The software architecture of the simulator facilitates this with a modular and flexible design.

The used design concepts are the Model-View-Controller concept, the Event System concept, and the Client-Server concept. The system is divided into one simulation server controlling the simulation and multiple clients computing different views, especially for the various truck mirrors (see Fig. 4). Communication is done by exchanging events over TCP/IP with the help of an event router. Server and clients are composed by specialized components. 


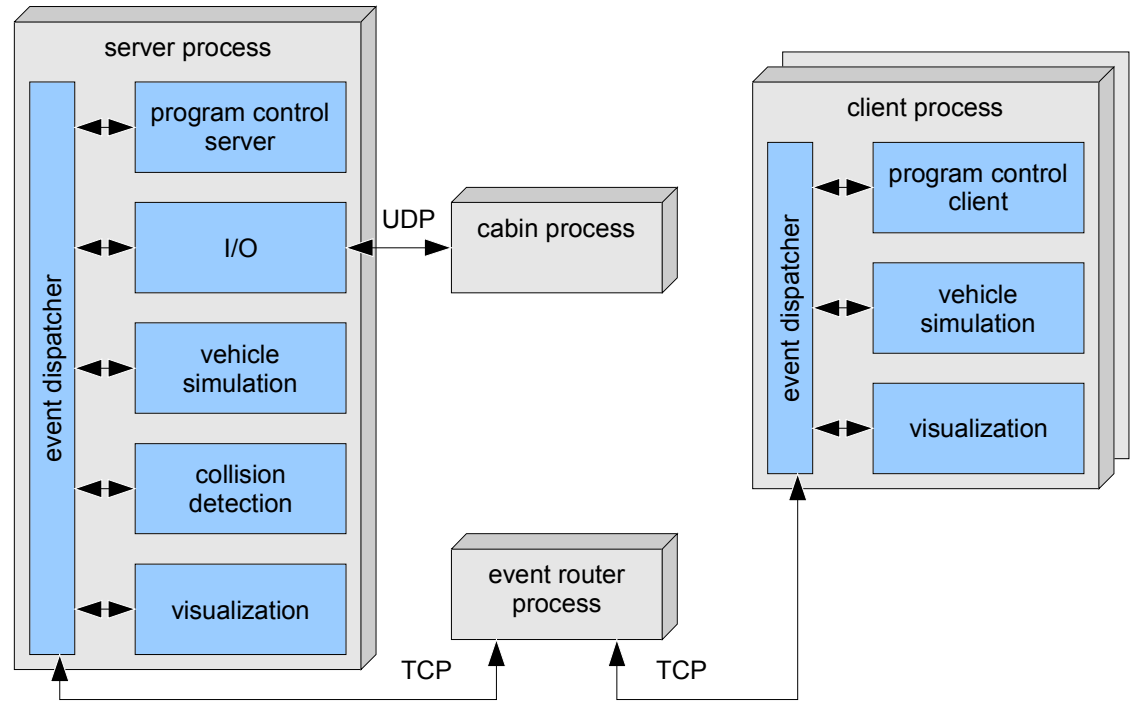

Fig. 4: Software architecture of the simulator.

The program control component controls user input, vehicle movements and visualization. It processes events generated by the $\mathrm{I} / \mathrm{O}$ component, which communicates with the simulator cabin or other hardware like keyboard, mouse or joystick. The controller uses the input events and further information like detected collisions to manipulate the vehicles, the visualization and the gages and indicators of the cabin.

The vehicle simulation component uses an abstract drive train model and the described kinematic model to calculate the vehicle movement based on the current vehicle state, throttle and gear. Because the input changes constantly and the vehicle moves only in a small distance between displayed frames, an iterative approach is used for the movement calculation. This also permits relatively simple movement calculations for complex vehicle configurations.

With the help of proxy classes, changes to the kinematic vehicle model automatically update the associated structural vehicle model (see Fig. 5). These updates mostly consist in positioning the overall structure and rotating and turning the wheel representatives. Glued to the structural model parts are the 3D model parts.

The visualization component uses an open source 3D engine

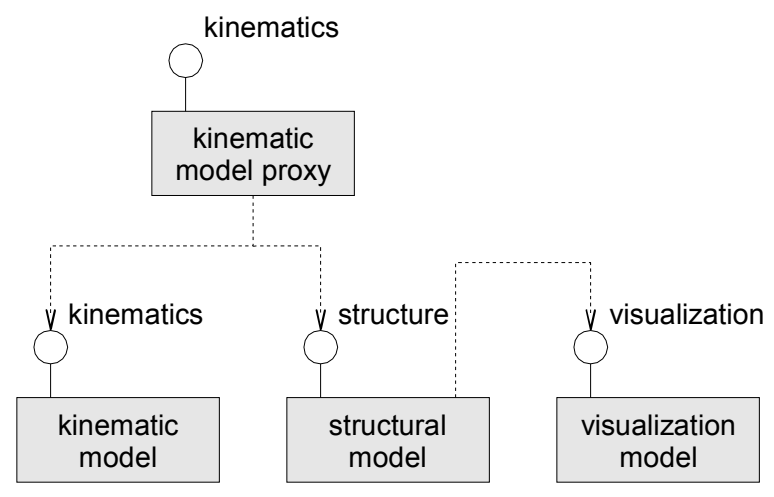

Fig. 5: Vehicle models. called CrystalSpace for rendering and displaying the vehicles and the environment. This engine can also be used to detect vehicle collisions based on the $3 \mathrm{D}$ model data.

For better display performance, the server and clients are executed on different computers. Because of fluctuating event transfer times, the simulation state known to server and clients differs. This is countered with program clock synchronization and time stamping the events. With this information, each client adjusts the vehicle position based on the last known server state and the local time. This leads to smooth animations on all systems even with different hardware and fluctuating CPU or net load.

Due to the modular conception, the simulator software can easily be adapted to different types of applications. Changes mostly affect the integration of new system components and the adaptation of the program controller. 


\section{Truck Driving Simulator}

The truck driving simulator is a commercial driving stand with original DaimlerChrysler truck parts like steering wheel, throttle, brake, handbrake, dashboard with electronic gages and semi automatic gear shift. The sight through the windshield is visualized by a video projector. In addition, an LCD-display is mounted at each side of the simulator to visualize the images of the left and right exterior mirrors (see Fig. 6). To increase the performance and the frame rate, the simulation software is distributed on three computers. The main computer is responsible for the computation of the sight through the windshield and the driver assistance systems. The other two computers are responsible for the left and right exterior mirror images. Both computers are linked to the main computer to get the current position and state of the articulated vehicle.

The main focus of the simulator is to learn and train to reverse commercial vehicles with trailers. In everyday life, the driver can be faced with different types of trucks and trailers, with different lengths and different kinds of steering behavior. To meet this requirement, the simulator comes up with a set of typical trucks and trailers like semi-trailer, one-axle trailer or two-axle trailer with single-pivot steering.

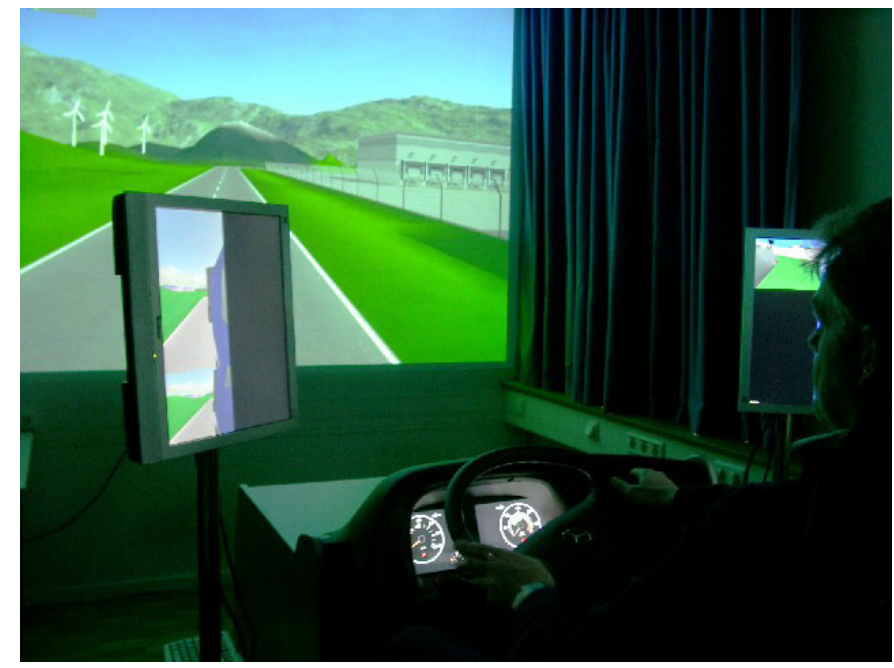

Fig. 6: Driving simulator in a field test.

Drivers of articulated vehicles are often faced with typical driving maneuvers like driving straight backwards, driving backwards through a left or right curve, or driving backwards to a loading ramp. Therefore, the 3D-simulation environment is especially constructed for this kind of driving maneuvers. The 3D-simulation environment consists of a haulage company ground and its immediate vicinity where all relevant maneuvers can be driven. Nevertheless, the simulator can be used for driving forward to learn the steering behavior of articulated vehicles in left and right curves for city driving as well ${ }^{(4)}$.

Figure 7 comprises the information visualized to the driver. In the middle of Fig. 7, the sight through the windshield is shown. In the left top of the windshield, the driver is supported by a visual steering assistance system. Based on the steering angle $\alpha$ and the bending angle $\gamma$, the outlines of truck and trailer are visualized together with two trajectories. The blue trajectory shows the track of truck and trailer in the case of a constant bending angle. The green trajectory shows the trailer tractrix curve. This tractrix curve represents the track the trailer will take on the next 20 meters. Based on the trajectories, the driver can imagine the future behavior of truck and trailer. 


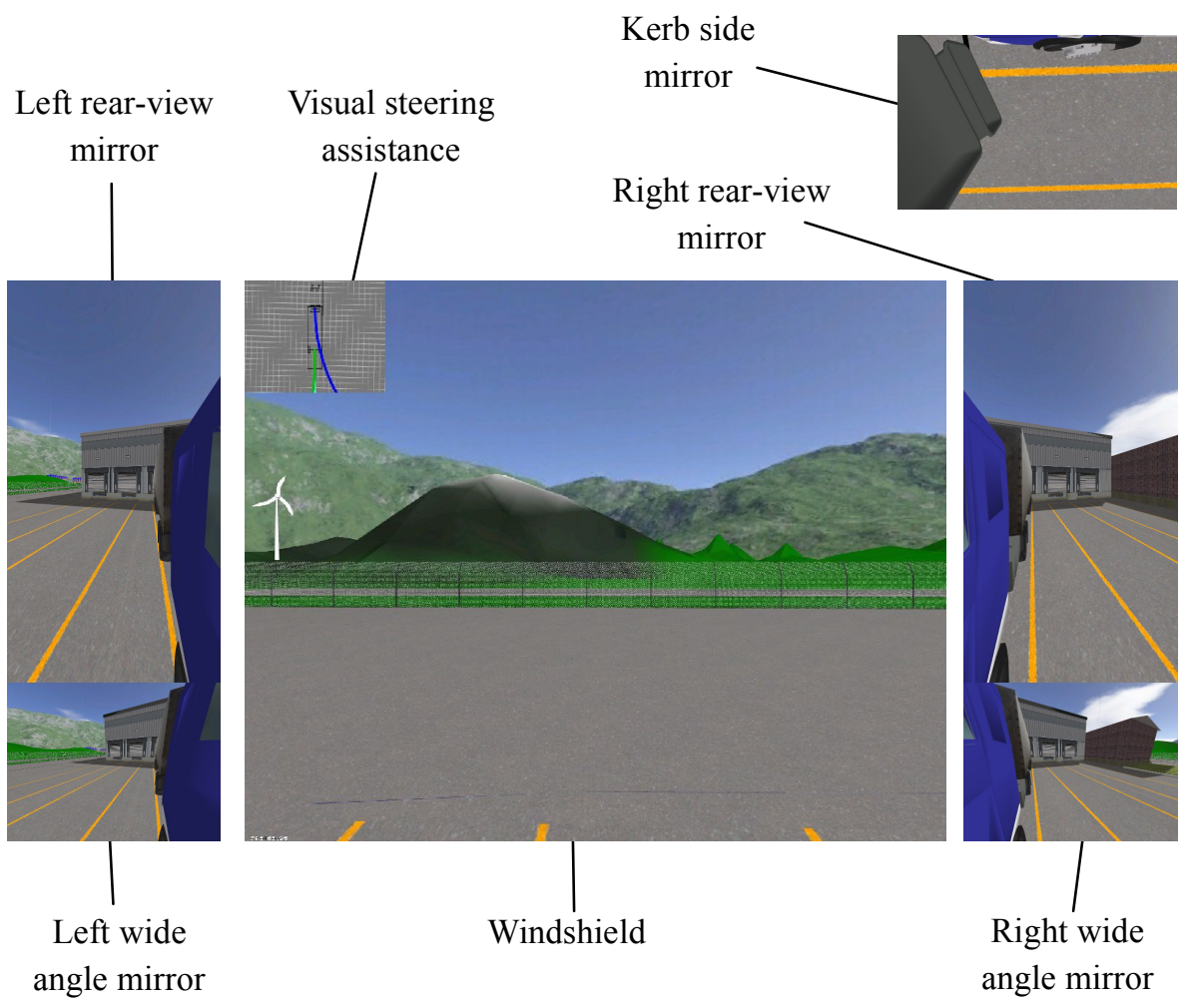

Fig. 7: 3D-simulation composed by the sight through the windshield and the left and right exterior mirrors.

Furthermore, the simulator computes the images of the exterior mirrors. The left and right exterior mirrors are both composed by a rear-view mirror and a wide angle mirror. Both wide angle mirrors are used to reduce the blind area beside a truck (see Fig. 1). The kerb side mirror in Fig. 7 shows the top down view along the right side of the driver's cab. At the top of the kerb side mirror the right front tire and the associated rim can be seen.

Additionally, we developed a low cost version of the described driving simulator. The virtual vehicles, the sight through the windshield, all exterior mirrors, and the driver assistance systems are altogether computed on just one computer (see Fig. 8). This version runs on a single computer with commercially available hardware. All information shown in Fig. 7 is visualized onto a 24-inch computer display. The virtual vehicles can be steered by joystick or steering wheel. The big advantage of the low

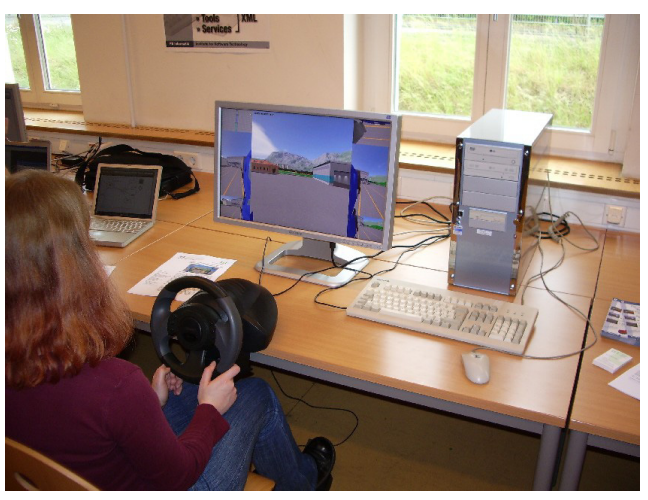

Fig. 8: Low cost driving simulator. cost version is, that everybody can learn and train reverse driving maneuvers of articulated vehicle at home at his/her own computer.

A realistic vehicle simulation should not be restricted to the driver visual perception channel. Especially in the field of vehicle and simulation development, the acoustics has a high significance. Only the combination of the acoustic and visual perception channel leads to a realistic vehicle simulation.

The acceptance of products is affected by the driver experiences and expectations. According to the driver expectations, it is very important to design a suitable acoustic scenario, because vehicle sounds communicate a multitude of relevant information for 
driver activities. During extensive interview studies, published in (9), drivers were asked about vehicle sounds which should not be absent in a vehicle. The result of the study is shown in Fig. 9. The most important sound is the motor sound.

"What kind of sound should not be absent in a vehicle?"

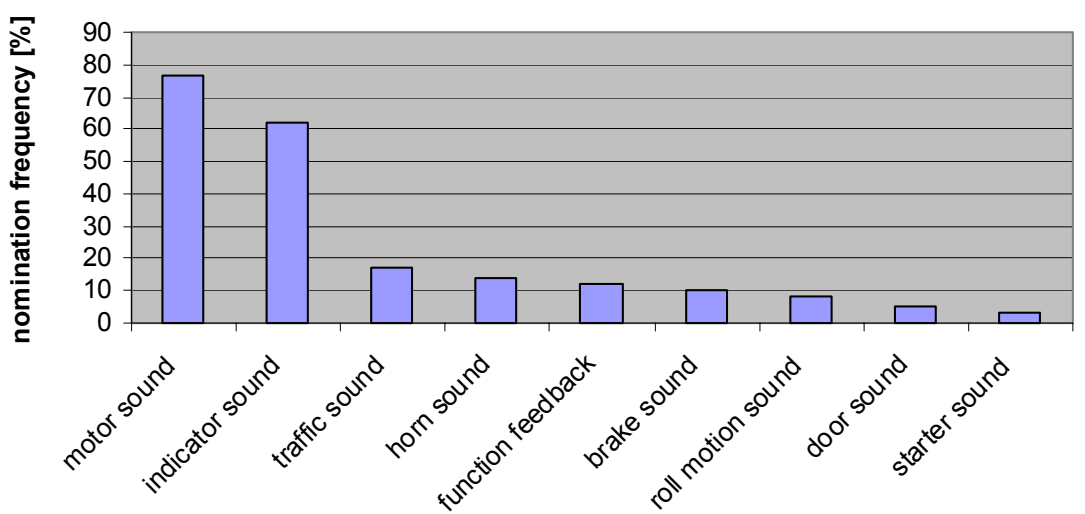

Fig. 9: Information requirements in relation to vehicle sounds ${ }^{(9)}$.

Therefore, we recorded the motor sounds of a real commercial vehicle and prepared the samples for our simulation environment. Based on these samples, the following activities are simulated:

- Ignition on/off

- Engine idle (750rpm)

- Engine with different revolution speed (1000rpm, 1500rpm, 2200rpm)

- Start and accelerate

- Impulse cutoff

- Gear change

- Air supplied brake

\section{Application Perspectives and Capabilities}

There are a number of application possibilities for the described driving simulator. The most important application is to learn and train the steering behavior of articulated vehicles like commercial vehicles with trailers or cars with caravan. Furthermore, the driver can learn and practice difficult driving maneuvers. During a number of interviews with professional truck drivers, we identified a set of driving maneuvers for backing up articulated vehicles which are said to be more or less difficult. The most important maneuvers out of this set are listed below in the order of an increasing degree of difficulty:

- Driving straight backwards

- Driving backwards in a (stable) left curve

- Driving straight backwards into a garage

- Driving backwards in a (stable) right curve

- Driving backwards in a left curve to a loading ramp

- Driving backwards in a right curve to a loading ramp

- Driving backwards into a parallel parking-gap

- Loading a swap trailer

The driver training starts with a short briefing about the simulator and its handling. Afterwards, the driver is faced with the allegedly easiest maneuvers. In dependency of the driver skills and the progress during the learning process, the demands on the drivers will increase steadily by more difficult driving maneuvers. When the driver is able to cope with 
the most of the listed maneuvers, some of them are composed to different scenarios the drivers are faced with during their daily work. In this way, the driving simulator may complement training, testing and licensing of commercial motor vehicle drivers ${ }^{(3)}$.

In the context of driver training, the simulator offers a wide range of advantages. One of the main advantages is the possibility to give more and better driving instructions. In safety critical situations for instance the driver is supported by warnings. In the case of a collision with other objects, the Fig. 10 (a) is shown on the windshield of our simulator. If the vehicle is driving over an obstacle like a kerb Fig. 10 (b) will appear on the windshield. Another critical situation is a too strong bending of the trailer. This situation may cause damages of truck or trailer. To warn against a possible accident, Fig. 10 (c) is shown on the windshield.

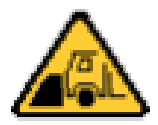

(a)

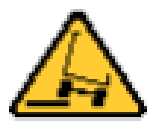

(b)

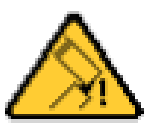

(c)

Fig. 10: Indication of critical situations.

Student drivers are often instilled to handle articulated vehicles by empirical formulae. These formulae give steering hints to control the vehicle in dependency of the respective truck and trailer, the driving maneuver and the position of the trailer into the rear-view mirrors. The simulator offers the possibility to learn and internalize these empirical formulae as well.

Another important advantage of the simulator is the reduction of driver training costs. By the use of the simulator, there is no necessity to hold an appropriate truck fleet with real vehicles in readiness. For covering special requirements, the simulator can be extended by creating new truck and trailer models. Moreover, mistakes of student drivers do not lead to real damages and costly repairs of vehicles. Altogether, the simulator is a cost-effective way to enhance the instruction and training quality.

Finally, we can visualize the driving scene from different points of view. In addition, it is possible to record the driving lesson for later evaluation or playback from different viewpoints. The recorded information is used to analyze the driver skills. Furthermore, a comparison between the desired and the actual vehicle position is useful for an objective appraisement whether the driver skills have been improved.

The flexible design of the software architecture offers the opportunity for other uses than just driver training. In our working group, the simulator is used for creating, testing and evaluating applications based on articulated vehicles. Among other things we develop steering assistance systems with visual, tactile and audio feedback to support drivers of articulated vehicles. These steering assistance systems are used in simulation environments, in a truck model or in a test vehicle. To ensure the usefulness of the realized steering assistance systems we accomplish systematic evaluations using the simulator ${ }^{(10)}$.

\section{Discussion and Outlook}

The use of driving simulators for backing up a vehicle or even an articulated vehicle is often said to be the most unreal experience that a driver can experience on a simulator. The chief difficulty is to estimate the distance to other objects or the dimensions of other objects. This problem is still not resolved in its entirety. Despite of the prevailing opinion, the use of the driving simulator as learning environment was successfully.

First, the simulator was tested within a special driver training of a German automobile association. The 8 participating novice drivers of articulated vehicles were taught to handle vehicles with one-axle trailer by empirical formulae. With the help of a half hour simulator training, all test persons were able to accomplish reverse driving maneuvers like driving 
straight backwards or backing up into a garage.

In addition, we carried out an evaluation of the driving simulator. Altogether, 20 test drivers participated, 5 driving instructors, 5 professional truck drivers and 10 car drivers with different experiences in backing up vehicles with trailer. All participants had to accomplish a set of 7 driving maneuvers with different vehicle types within 2 hours.

At first, we had a look at the important question of simulator sickness. Many simulator manufacturers are confronted with the problem of simulator sickness. Generally, it is said, that up to $30 \%$ of all simulator test drivers suffer more or less from simulator sickness. After the two hour test, just one test driver was suffering from light headache. While driving forward with a velocity between 20 and $60 \mathrm{~km} / \mathrm{h}, 3$ test persons got a bit dizzy.

With the experience of the evaluation, $80 \%$ of the driving instructors and $100 \%$ of the professional truck drivers recommended to use the computer based simulator training to instruct student drivers. The simulator would offer the opportunity to learn and internalize empirical formulae. Moreover, the simulator reduces the wear out of vehicle parts like tires and gear box and does not pollute the environment.

In addition to the use for driver training, the simulator offers a wide range of further application possibilities. Due to the flexible and modular conception, the simulator can be used for creating, testing and evaluating different kinds of applications based on articulated vehicles. Therefore, we are going to use the simulator for a systematic evaluation of our steering assistance systems as well. The use of the simulator leads to a reduced risk of damages to persons or vehicles. Moreover, there are always the same environmental conditions in the virtual environment. This leads to a good comparability of the test results.

Furthermore, the simulator can be extended in various ways. In the case of difficult driving maneuvers, the ideal trajectory can be given by the simulation. The student driver can try to follow the proposed lane and internalize the required steering input. In addition, the driving maneuvers can be executed automatically by the simulation to instruct the student driver how to handle the articulated vehicle. In a further step, the hints can be reduced gradually until the driver is able to cope with the articulated vehicle without any additional help ${ }^{(11)}$.

\section{References}

(1) Zöbel, D. and Balcerak, E., Precise driving maneuvers for articulated vehicles (in German), Proceedings of Autonomous Mobile Systems 2000 (AMS 2000) (in German), pp. 148-156.

(2) Zöbel, D., Polock, D., and Wojke, P., Steering assistance for backing up articulated vehicles. Proceedings of International Conference on Computer, Communication and Control Technologies (CCCT'03), vol. I, 2003, pp. 199-204.

(3) Whitmire, J., Tarr, R. W., and Allen, T. L., Tractor-Trailer Simulation and the Assessment of Training Scenarios for City Driving: Skill Building in the Area of Left and Right Turns, Proceedings of the Driving Simulator Conference North America, 2005.

(4) Berg, U. and Zöbel, D., Haptic steering assistance for backing up vehicles with one-axle trailer (in German), Proceedings of 6. Berlin Working Committee Men-Machine-Systems (BWMMS 2005) (in German), Vol. 22, No. 19, 2005, pp. 131-136.

(5) Zöbel, D., Schikora, J., and Wojke, P., Minimalized driver assistance for trailer backup, Proceedings of International Conference on Computer, Communication and Control Technologies (CCCT'04), volume IV, 2004, pp. 347-352.

(6) Chang, J. S., Lin, J. H., and Chiueh, T. D., Neural networks for truck backer-upper control system. Proceedings of the International IEEE/IAS Conference on Industrial Automation and Control: Emerging Technologies, 1995, pp 328-334.

(7) Kong, Seong-Gon and Kosko, Bart, Comparison of Fuzzy and Neural Truck Backer-Upper Control Systems, chapter 9, 1992, pp 339-361, Prentice Hall International. 
(8) Schoenauer, Marc and Ronald, Edmund, Neuro-genetic truck backer-upper controller, Proceedings of the First International Conference on Evolutionary Computation (ICEC94), 1994.

(9) Beier, G., Boemak, N., and Renner, G., Sense and sensuousness - psychological contributions to vehicle design and appraisal (in German), Jürgensohn, T. and Timpe, K.-P., Vehicle controlling (in German), 2001, pp. 263-284.

(10) Theimert, C. and Negele, H.-J., Evaluation of driver assistance systems on driving simulators in the industry of commercial vehicles (in German), Integrated Safety and Driver Assistance Systems (in German), Vol. 1864, 2004, pp 61-77.

(11) Allen, T. and Tarr, R., Driving simulators for commercial truck drivers - humans in the loop, Proceedings of the $3^{\text {rd }}$ International Driving Symposium on Human Factors in Driver Assessment, Training, and Vehicle Design, 2005. 